\title{
EDITORIAL
}

\section{Preface for Theory and Clinical Practice in Pediatrics}

\author{
Editor-in-Chief: Giulio Filippo Tarro
}

\section{Preface}

Pediatrics is the branch of medical science that deals with growing development, mental health and disease prevention from fetus to adolescents. With severe global environmental problem, virus mutation, growing cases of children cancer, the emerging advanced methods of examination, diagnosis and therapy (genetic test, new vaccine, precision medicine, metabonomics, molecular imaging, genetic therapy, minimally invasive therapy and interventional therapy, etc.), new theories and methods have sprung up in the field of pediatrics clinical research. The emerging diseases and the significant importance of research aimed at discovering new methods for improving diagnosis and therapy keep the topic of microbe involvement and vaccines among the most interesting and urgent problems of medical science today. It is, therefore, an honor for me and a great privilege to lead a new journal Theory and Clinical Practice in Pediatrics.

The publisher strongly believes in making research readily available to everyone and at the same time wants to provide always ethical services to the readers. Our journal would like to provide a platform for pediatrics researchers and related researchers to share new theories and methods.

Theory and Clinical Practice in Pediatrics is a broad ranging, international peer reviewed journal to pediatrics, publishing theoretical, clinical, and professional practice issues relevant to pediatrics, as broadly defined.

The scope of the journal includes, but not limited to, allergy and immunology, cardiology, critical care, emergency medicine, endocrinology, gastroenterology, hematology, infectious disease, nephrology, neuropsychology, oncology, pulmonology, genetics, neonatology, developmentalbehavioral medicine. The journal has the potential to flourish and progress following the international publication and academic guidelines.

The papers to be selected shall cover a wide range and encompass the most up-to-date information on the multifactorial events which lead to potential pathologies in the world.

I would like to express my sincere thanks to the members of SyncSci Publishing Pte Ltd and to all who contribute to their participation, help and interest in the success of the journal. The knowledge that we yield today concerning the involvement in pediatric diseases represents one of the most important advancements of human health. 